\title{
STUDY OF THE TEVATRON NONLINEAR \\ ACCEPTANCE BY COMPUTER SIMULATION
}

F. Willeke

November 1,1983 
F. Willeke

Study of the TEVATRON Nonlinear Acceptance

by Computer Simulation

(1) Introduction

In this report, the results of computer calculations of nonlinear acceptance reduction caused by nonlinear fields in the TEVATRON are presented. A set of test particles has been traced during many revolutions in the accelerator in order to determine the maximum stable amplitude in the presence of nonlinear fields. Sources of nonlinear fields are designed imperfections of dipole and quadrupole magnets, fluctuations due to manufactoring tolerances, and sextupole fields needed to compensate for chromaticity.

The acceptance is a property of the linear machine. It is defined as the area of the phase ellipse given by the coordinates of the particle with the maximum stable betatron amplitude restricted by the physical aperture. If nonlinear forces act on the particle the phase area is no longer an ellipse. In this case the acceptance may be defined as the maximum phase ellipse which remains within the boundary of the maximum phase area deformed by nonlinear fields. This is useful as long as the nonlinear forces are small perturbations of the linear ones. However if the physical aperture becomes as large as the the dynamical aperture, which is defined by the separatrix of the nonlinear phase area for infinite physical aperture, the acceptance defined in this way may depend on the position in the lattice, where it is evaluated $/ 1 /$. In this case one may define the acceptance as 
the minimum one which can be found in the lattice. This definition is used in this report and should be kept in mind when the results are compared with other calculations or measurements. The aim of the study is twofold:

An actual question of practical interest is how much acceptance is available for the low beta optics (for the colliding beams), where the linear acceptance is reduced by a factor of about 4 and the natural chromaticity is increased by $50 \%$ compared with the high beta optics (present status for extraction). In the low beta case, multipole fields in the quadrupoles of the low beta insert, where beta functions exceed almost 1000 m may become important. Because stronger sextupole focussing is needed in addition, a stronger nonlinear reduction of acceptance than in the extraction optics may occur. In order to investigate this problem, it appears to be useful to compare the results of tracking calculations for the low beta case with the results for the extraction optics. Tracking results for the extraction optics can now be checked by measurements on the TEVATRON.

The other motivation of this study is the possibility to extrapolate the experimentally verified tracking calculations of the first superconducting accelerator to future accelerators. If it will be possible to verify tracking calculations based on measured multipole components of TEVATRON magnets by measuring TEVATRON acceptances one will get more confidence to use tracking results as a criterion in order to determine field quality and aperture. For this purpose, calculations with the measured multipole field components of each individual dipole and quadrupole mag- 
net are compared with calculations based on random fluctuations about the mean values of multipole components. This is not the only tracking study for the TEVATRON. Similar calculations with comparable computer codes are being done with somewhat different emphasize /2/. It will increase confidence in computer tracking if one compares those results with the ones presented here and finds agreement.

\section{(2) Procedure}

The tracking has been performed by using the computer code RACETRACK /3/. In order to save computing time, successive pure linear lattice elements are combined in a linear block. These blocks are separated by nonlinear elements, which are treated in the thin lens approximation. For the statistical multipole distributions, only one nonlinear element situated in the middle of the TEVATRON half cell is assumed which contains the integrated multipole fields of the four individual dipole magnets.

However it has been verified, that results don't depend significantly on the position of the nonlinear element in the half cell, so that this approximation which saves $75 \%$ computing time is justified. Multipole components of quadrupole fields have been neglected for the extraction optics and are taken into account only in the BO long straight section quadrupoles in the low beta optics. Because no data for the iron magnets which are part of the abort system in the co straight section are available, the same multipole fields as for the superconducting magnets are used. The latter assumption should have negligible impact on the results. 
Individual multipole fields are assumed to be gaussian distributed about the mean value of the corresponding component. The distribution is cut beyond the 5 th standard deviation. The mean value of the quadrupole component of the bending field can be compensated almost exactly by means of the existing correction quadrupoles in each cell. It has therefore been neglected in the tracking calculations. The rms and mean values result from systematic measurements on each of the TEVATRON

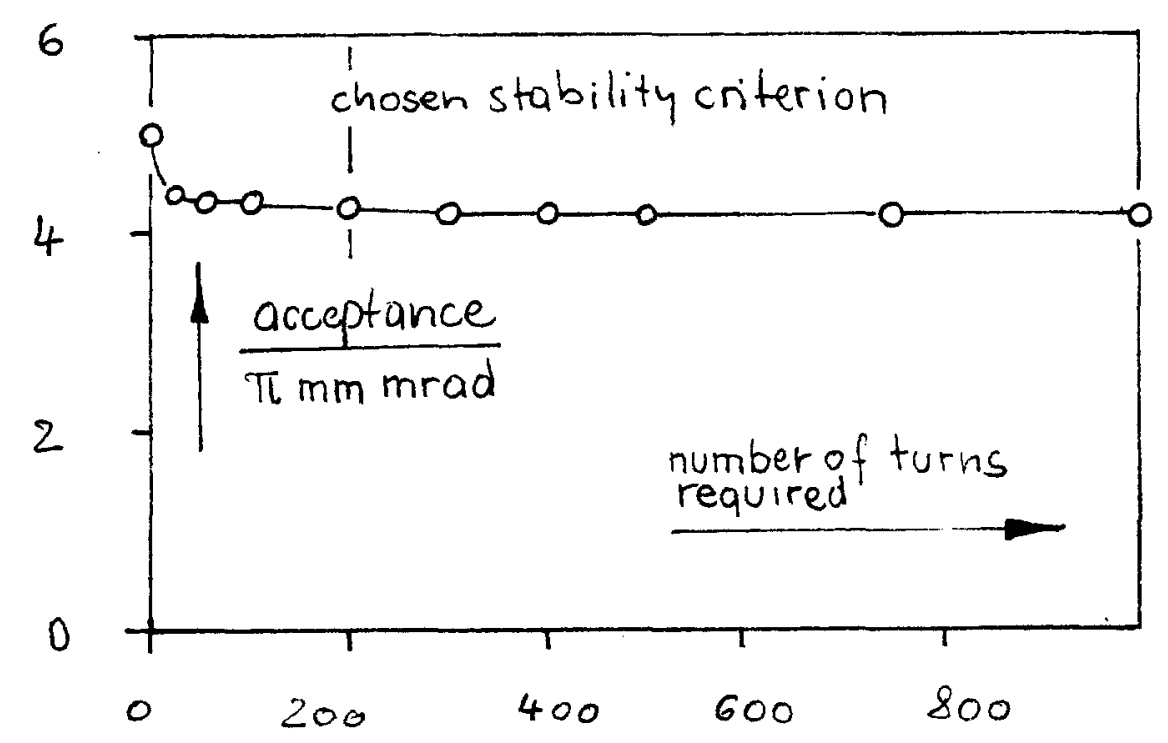

fig 1 Acceptance as Given by RACETRACK Plotted Against Required Number of Stable Revolutions

superconducting magnets /5/. The mean and rms values of measured multipole components as used for the calculations are listed in tables 1 and 2 . 


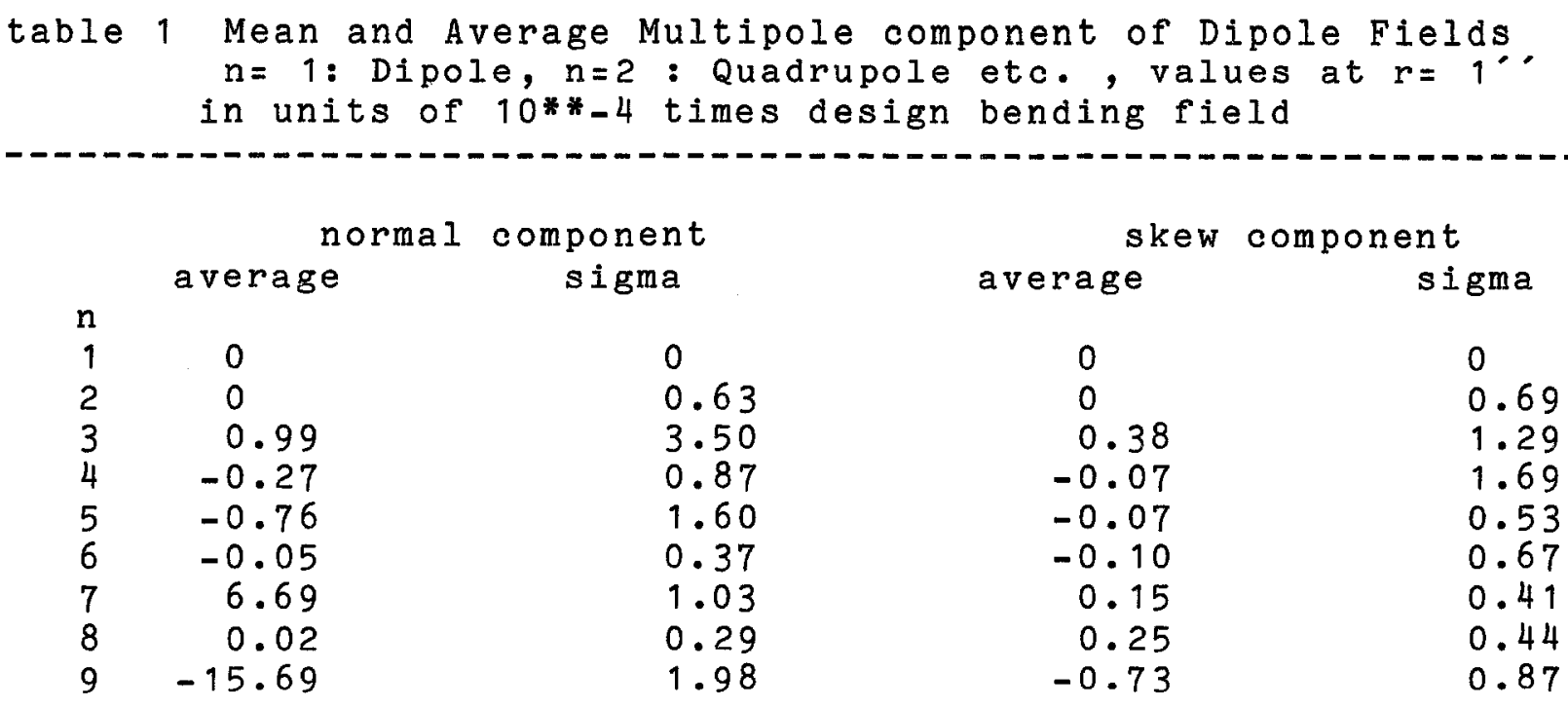

\section{table 2 Mean and Average Multipole Component of Quadrupole Fields $n=2$ : quadrupole, $n=3$ : sextupole etc., values at $r=1 \ldots$ in units of $10 * *-4$ times design bending field}

\begin{tabular}{|c|c|c|c|c|}
\hline & \multicolumn{2}{|c|}{ normal component } & \multicolumn{2}{|c|}{ skew component } \\
\hline & average & sigma & average & sigma \\
\hline \multicolumn{5}{|c|}{ 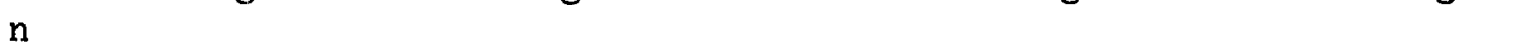 } \\
\hline 1 & 0 & 0 & 0 & 0 \\
\hline 2 & 0 & 0 & 0 & 0 \\
\hline 3 & 2.29 & 3.90 & 3.07 & 3.78 \\
\hline 4 & 1.28 & 0.97 & -0.40 & 1.97 \\
\hline 5 & -0.29 & 0.75 & -0.62 & 0.74 \\
\hline 6 & -1.89 & 1.45 & 0.21 & 0.38 \\
\hline 7 & 0.04 & 0.27 & 0.17 & 0.26 \\
\hline 8 & 0.04 & 0.18 & -0.01 & 0.19 \\
\hline 9 & -0.04 & 0.24 & -0.11 & 0.22 \\
\hline
\end{tabular}

Calculations for the TEVATRON 'as built' are performed for the extraction optics only. Here all measured multipole fields are taken into account as thin lenses in the middle of each dipole ( 4 per half cell) and each quadrupole up to the 12-pole component. For higher components, in all individual dipoles (or quadrupoles) 
the same average values are used.

The acceptance is determined by increasing the amplitudes of test particles until the first particle hits the physical aperture limitations within a given number of revolutions. The starting point on which the acceptance is evaluated is AO. It is midway between the points of maximum horizontal and vertical lattice functions in the ring without intervening nonlinear elements. This starting point leads to minimum values of acceptance. Starting at co the acceptances found for one particular case differ by $+20 \%$ from the AO results. The acceptances evaluated at AO don't depend on the number of required stable revolutions $n$ when $n$ is larger than $\mathrm{n}=100$ (see fig 1 ). The stability criterion has been chosen as $n=200$. This appears to be a good compromise between certainity of results and economic computing time consumption. The acceptance is limited in the TEVATRON quadrupoles where maximum beta functions occur. The quadrupole physical aperture is $35 \mathrm{~mm}$. The tracking has been performed for constant energy only. However the results are valid for the case of synchrotron oscillations too because the TEVATRON synchrotron oscillation period is about 3.5 times larger than the time particles are traced $\left(Q_{s}=0.0013\right)$. Two kinds of initial conditions have been used. In the very conservative case particles are assumed to have maximum betatron amplitudes in the horizontal and in the vertical plane as well (rectangular beam shape). In this case 16 particles are sufficient. In the more realistic case, particles are distributed on the surface of the four dimensional phase ellipsoid. Then 64 particles are needed to occupy sufficiently the initial phase area. The same values have been taken for maximum amplitudes in both directions. $\varepsilon_{x}=\varepsilon_{y}$, 'round beam'). 
For the extraction optics, the existing TEVATRON structure and usual optics parameters are used. For the low beta case, the lattice and Iinear optics parameters as presented by D.E. Johnson $/ 4 /$ is used for all tracking calculations. The beta functions at interaction point ( $B O$ ) are $\beta_{x}^{*}=80 \mathrm{~cm}$ and $\beta_{y}^{*}=85 \mathrm{~cm}$ (these values vary within $10 \%$ when the tune is changed by means of the correction quadrupoles contained in each TEVATRON half cell by about $+/-0.05$ ). The corresponding natural chromaticities are about -29 for both directions.

Before tracking, the linear chromaticity is compensated with two sextupole families. The tunes are adjusted near the usual TEVATRON working point with $Q_{x}=19.44$ and $Q_{y}=19.38$ for the extraction optics. For the low beta case they have been chosen as $Q_{x}=19.565$ and $Q_{y}=19.580$ in order to study the effect of nonlinear resonances. These adjustments are applied for the on energy optics only. After tune and chromaticity correction, the off energy closed orbit is determined for each energy. The linear optics is calculated on the closed orbit with the linear terms contributed by nonlinear elements taken into account.

\section{(3) Results}

a) High Beta (Extraction) Optics

Tracking results for the extraction case are summarized in fig 2,3 and 4 . The acceptances obtained from the tracking calculations are plotted as a function of the energy deviation dE/E. The solid line shows the linear acceptance. In the energy range of $\quad-0.30 \% \leq d E / E \leq 0.30 \%$ the acceptance is Iimited 
by the physical aperture in the extraction long straight sections A and D. For larger energy deviations it is limited at the maximum dispersion points in the arcs. The maximum linear acceptance is 5.0 II $\mathrm{mm}$ mrad. The maximum energy range is given by $-0.57 \%<d E / E<0.57 \%$. Calculations for a rectangular beam show a reduction of acceptance by $12 \%$ due to sextupole fields for
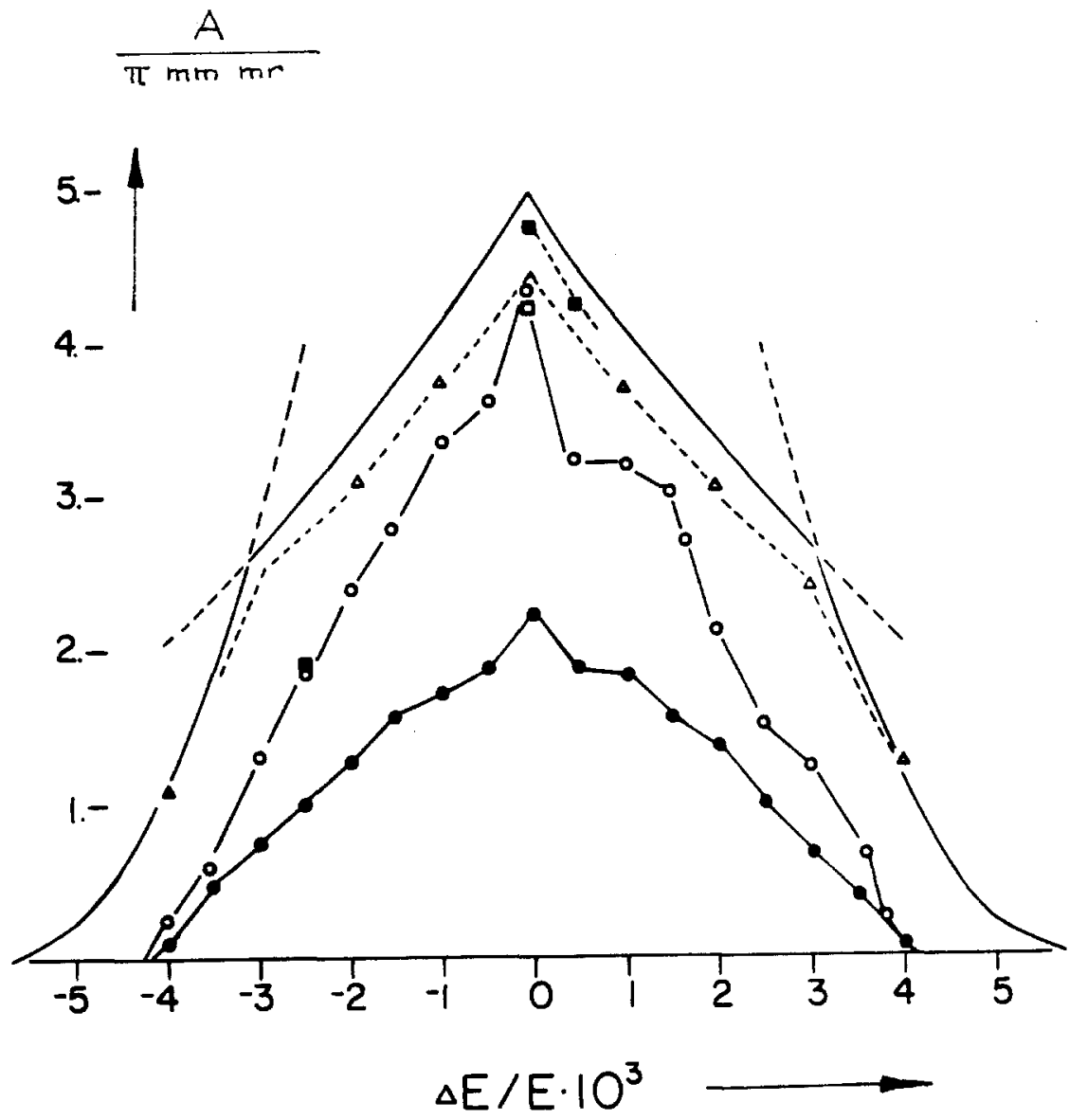

fig 2 TEVATRON Acceptance as a Function of Energy Deviation dE/E - Linear Acceptance (extrapolated from on energy optics)

$\Delta \quad$ Accceptance with Sextupole Fields (rectangular beam)

- with Averaged Multipole Fields, Horizontal Oscillations only

口 with Averaged Multipole Fields, Vertical oscillations only

O dito, Oscillations in both Planes, Elliptical Beam

- dito, Oscillations in Both Planes, Rectangular beam

chromaticity correction for small energy deviations ( triangles in 
fig 2 ). Corresponding acceptances for an elliptical beam differ only very little. Squares and circles indicate acceptances when average values of multipole fields are taken into account. In order to compare the results with those of the low beta optics, the high field measured values ( $I=4000 \mathrm{~A})$ of multipole components have been chosen. If one allows only horizontal betatron oscillations in the calculations the nonlinear acceptance reduction is only $5 \%$ at zero energy deviation but becomes larger at $\mathrm{dE} / \mathrm{E}=-.25 \%$ where nonlinear acceptance differs by a factor of 0.64 from the linear one (full squares fig 2 ). In the case of only vertical oscillations, the acceptance reduction is somewhat larger on energy (-15\%). Circles show results for oscillations in both planes. Whereas the calculations for an elliptical beam give approximately the same results as single plane oscillations (open circles), the acceptance is drastically reduced for a rectangular beam (by 50\% with respect to the elliptical beam case and by $56 \%$ with respect to the linear acceptance at $d E / E=0$ ). Taking into account fluctuations of multipole fields, the acceptance is reduced by another factor of 0.57 in the worst of five different statistical configurations ( see fig $3 a$ ). The systematic off energy explorations have been performed for a rectangular beam only. The maximum allowed betatron amplitude in the arc according to this nonlinear acceptance is $11 \mathrm{~mm}$. For an elliptical beam only the worst statistical case is calculated. Results are shown in fig 4 . On energy one derives a maximum amplitude of $x=15.8 \mathrm{~mm}$. For $\mathrm{dE} / \mathrm{E}=0.25 \%$ one finds $5 \mathrm{~mm}$ for the rectangular and $8.0 \mathrm{~mm}$ for the elliptical beam. These results have to be compared with those for the TEVATRON 'as built" . Calculations are carried out with elliptical beam. In fig 
4, acceptances are plotted together with average field acceptances and the worst statistical case for comparison. On energy one finds a maximum stable amplitude of $x=18.3 \mathrm{~mm}$ in the arc.

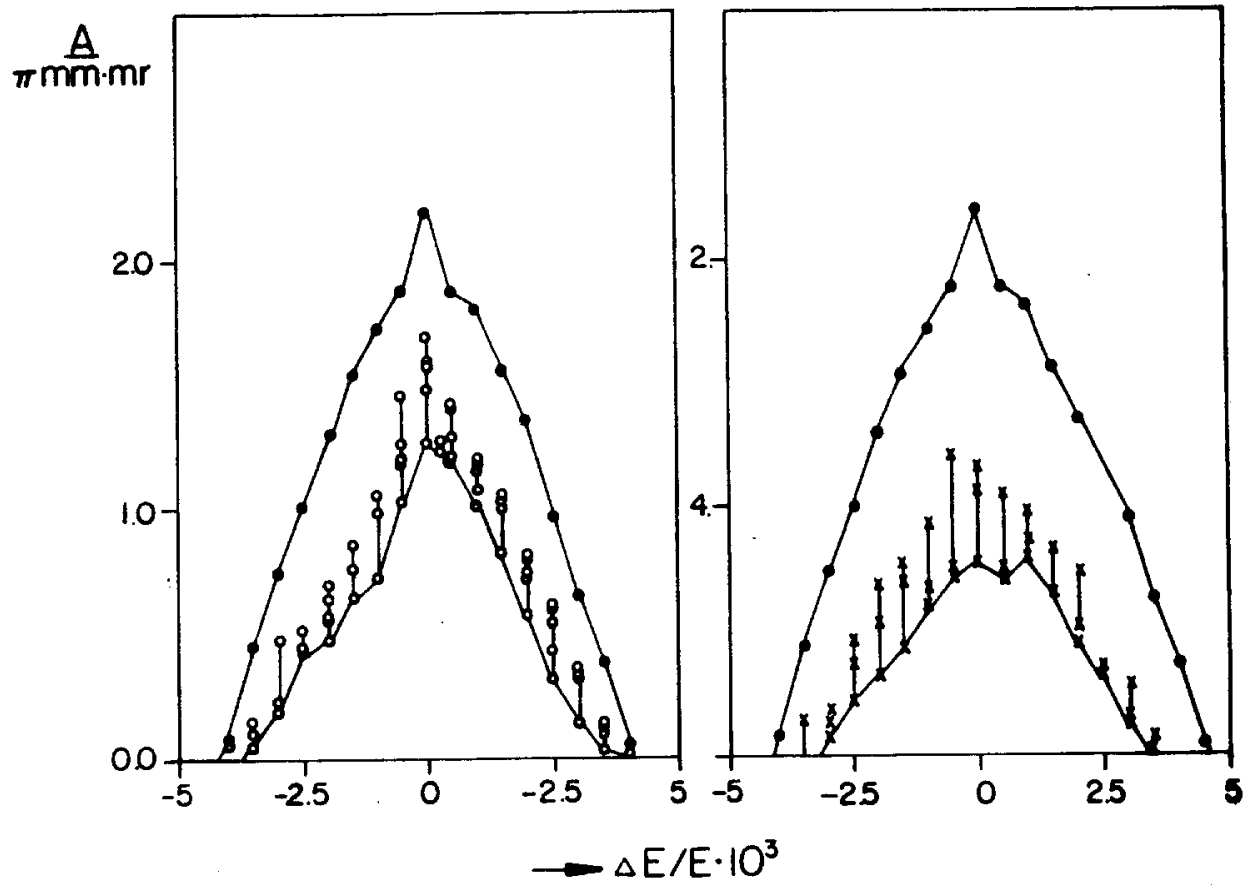

fig 3 TEVATRON Acceptance, Influence of Fluctuations

- with Averaged Multipole Fields for Comparison

- with Statistical Fluctuations of Multipol Fields

* Including Orbit Distortions $x-r m s=y-r m s=2 m m$

All Calculations with Rectangular Beam

Fig 3b shows additional acceptance reductions due to orbit displacements of $2 \mathrm{~mm}$ rms-values for both horizontal and vertical

orbit. Different points for same energy deviation correnpond to different statistical configurations. 


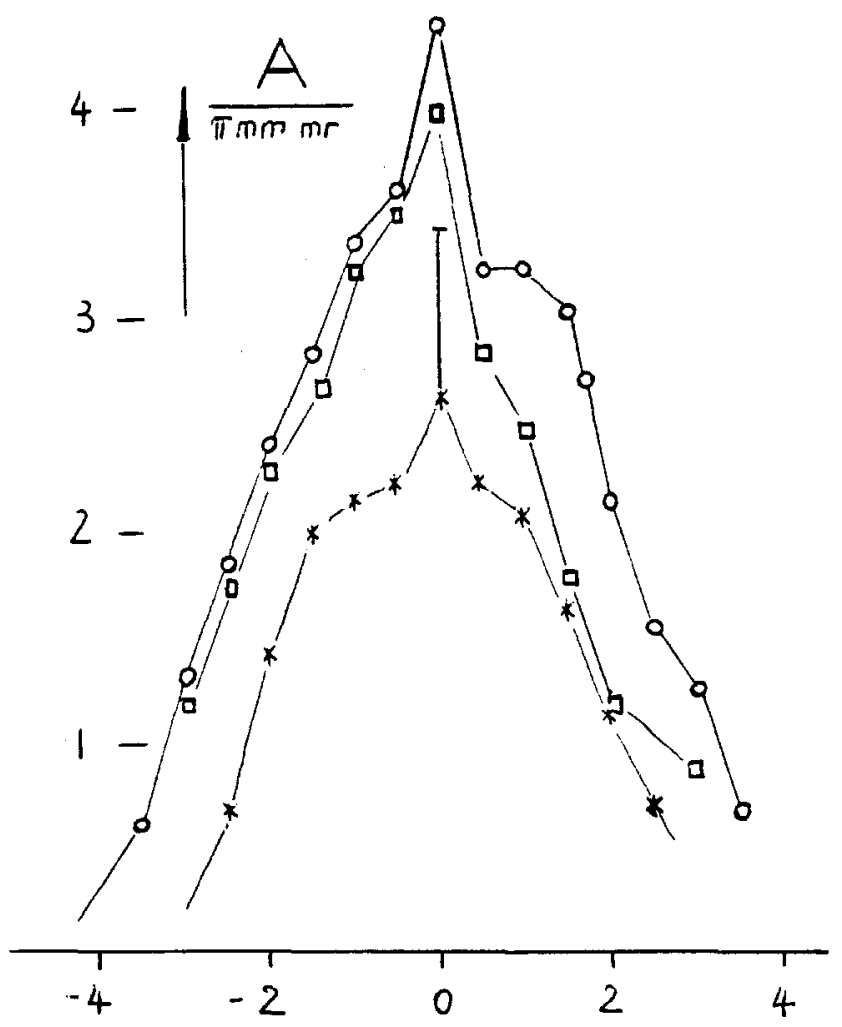

fig 4 TEVATRON Acceptance with all Measured Multipole Fields in Dipole and Quadrupole Magnets ("as built"))

a "as built.

- Average Values of Multipole Fields

* Worst of Five Statistical Multipole Configurations

b) Low Beta Optics (colliding beam case)

The results for the low beta optics are shown in figure 5. The solid line in $f$ ig 5 is the linear acceptance.

Triangles in fig 5 indicate sextupole acceptance. The sextupole fields are adjusted to compensate for the natural chromaticities. Nonlinear reductions of acceptance due to sextupole fields don't occur. The nonlinear acceptance is represented by circles in fig 5 . The nonlinear retuction amounts $17 \%$ on energy with respect to the linear acceptance. As in the extraction optics case it becoines considerably larger of energy. 
As for the extraction optics five different statistical multipole configurations have been evaluated in order to investigate the influence of fluctuations ( dots in fig 5, elliptical beam). The worst case gives $A=0.74 \pi \mathrm{mm} \mathrm{mrad}$. This corresponds to $8.4 \mathrm{~mm}$ free space for betatron oscillations and orbit displacements on energy in the arcs. In the large beta quadrupoles Q99 near BO there are still 26.3 mm free space available nevertheless.

\section{(3) Discussion}

In order to understand these results, they are discussed in detail below.

First the extraction optics will be investigated: For an elliptical beam no reduction of the acceptance is obtained when the sextupole fields are turned on to compensate for chromaticity. A small reduction is found for the rectangular beam, where the occupied phase volume has edges in the $x-y$ plane and maximum squared radii are twice as large (triangles in fig 2). One may conclude that chromatic effects have only negligible influence on TEVATRON acceptance as it is expected from the favorable ratio of chromaticities $\left(\zeta_{x i y} \simeq-22\right)$ to dispersion $D_{x}\left(D_{x}^{\max } \simeq 6 \mathrm{~m}\right)$. In this section, the influence of multipole fields will be discussed. After turning on the average multipole fields, horizontal focussing sextupoles have to be reduced by a factor 0.38 whereas the vertical focussing sextupoles have to be increased by a factor 1.59 to keep zero chromaticity.

The difference between horizontal and vertical acceptance on energy $(A=4.76 \pi \mathrm{mm} \mathrm{mrad}$ and $\mathrm{A}=4.22 \pi \mathrm{mm} \mathrm{mrad})$ is quantitati- 


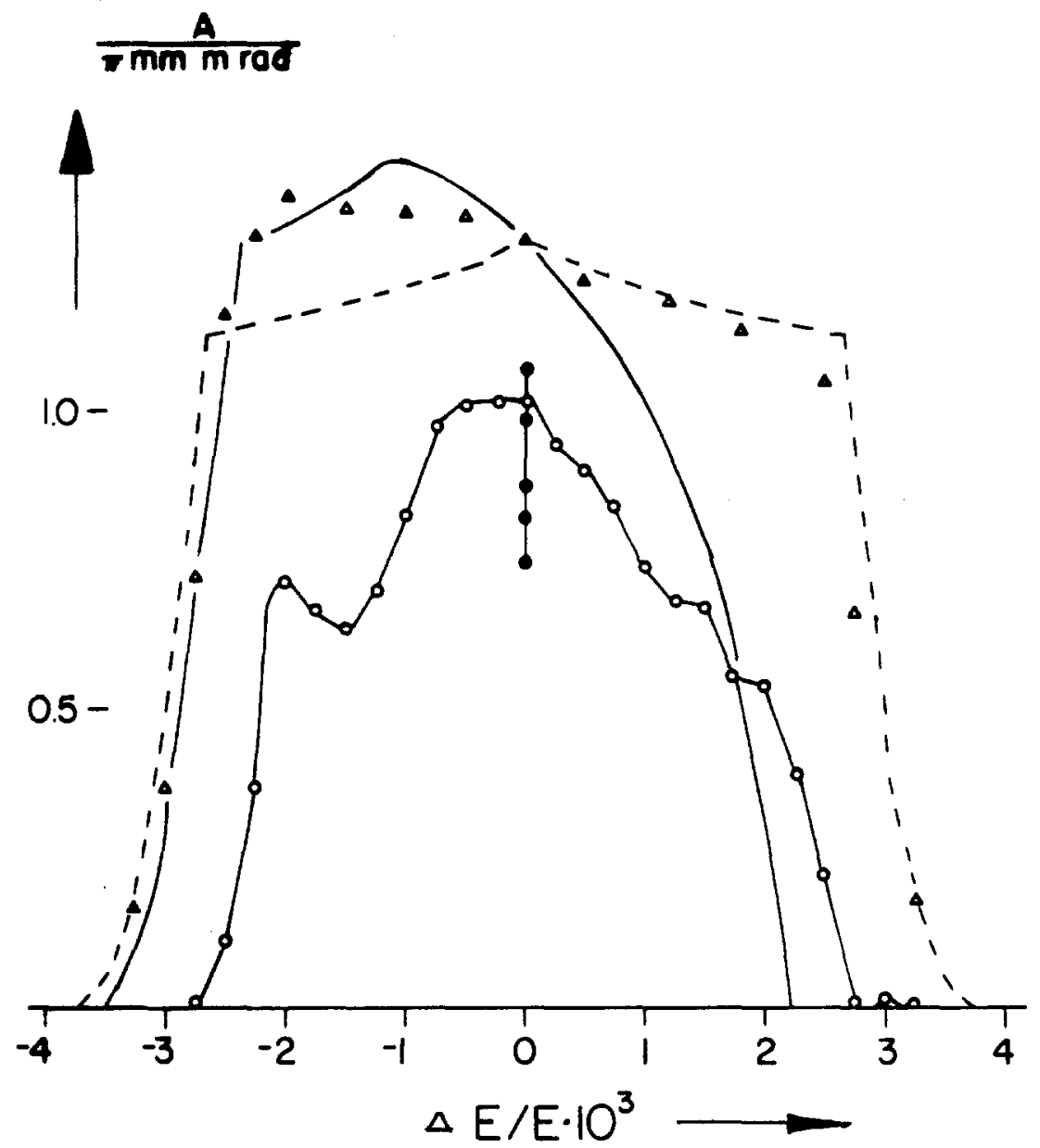

fig 5 TEVATRON Acceptance for Low Beta Optics

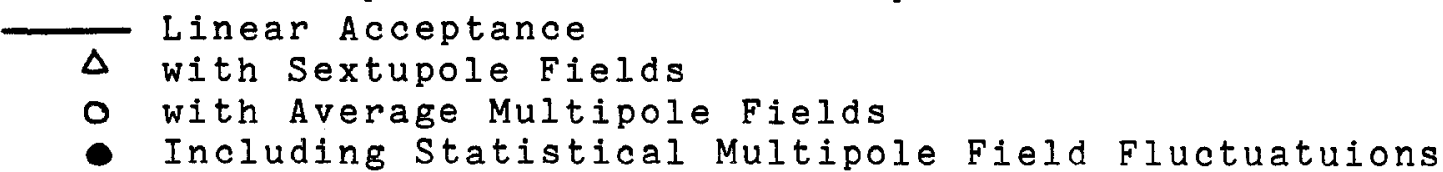

vely explained by the fact that the bending field drops earlier on the vertical axis than on the horizontal one.

The calculations for the rectangular beam are clearly too pessimistic.

This follows last not least from the fact, that in the real machine 
even larger amplitudes have been observed to be stable/6/. The fact that the acceptance for a rectangular beam differs by almost a factor of $1 / 2(0.52)$ from the acceptance of the elliptical beam indicates that the physical aperture is as large as the dynamic aperture. This can be demonstrated by calculating the acceptance for different physical apertures. For this purpose the worst of the statistical multipole configurations has been chosen, which may give a lower limit of dynamic aperture. Results are shown in fig 6. The maximum stable amplitude is plotted versus the physical aperture. The TEVATRON dynamic aperture is found to be $25.6 \mathrm{~mm}$.

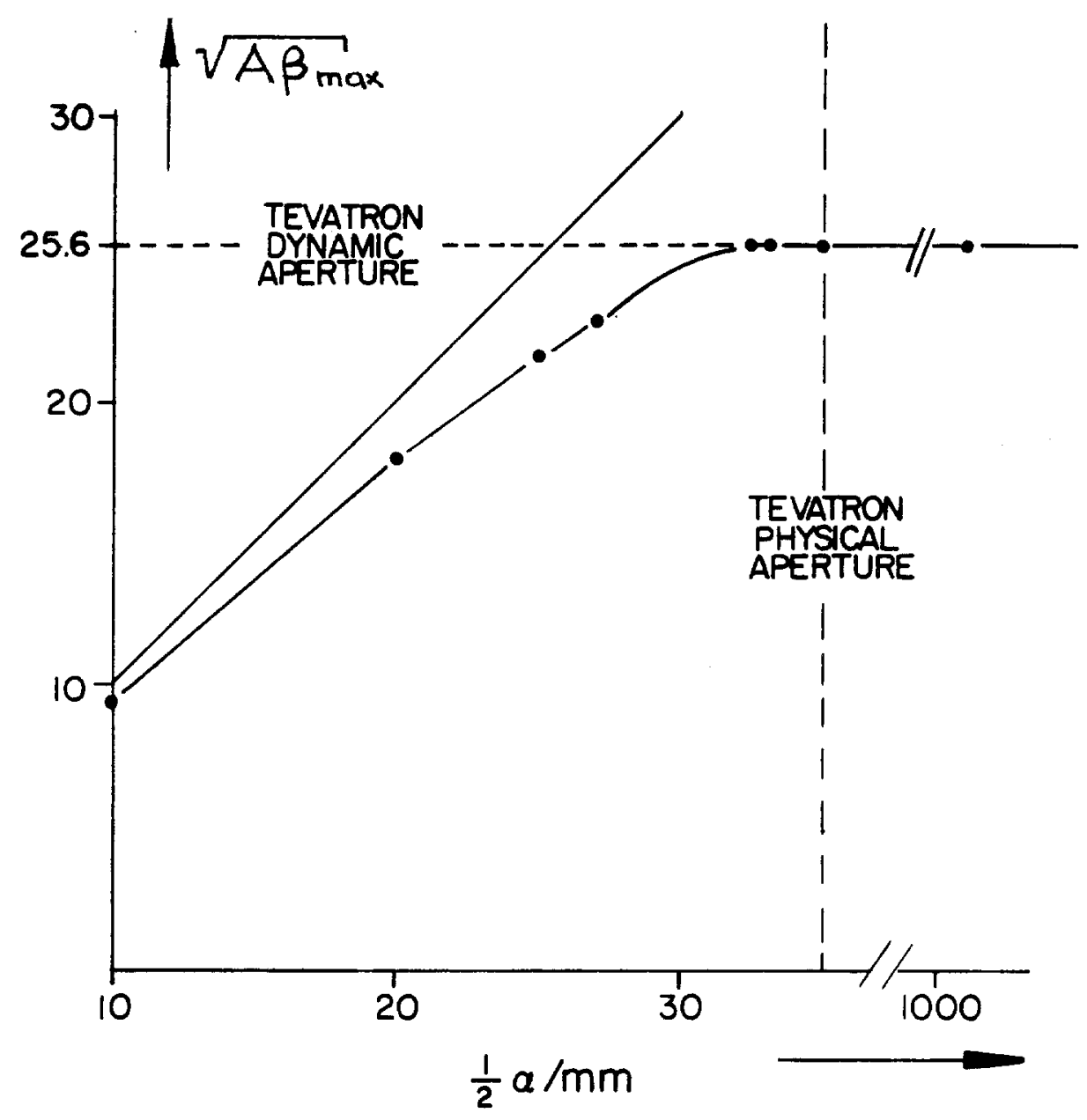

fig 6 TEVATRON Dynamic Aperture, Maximum Stable Amplitude as a Function of Physical Aperture 


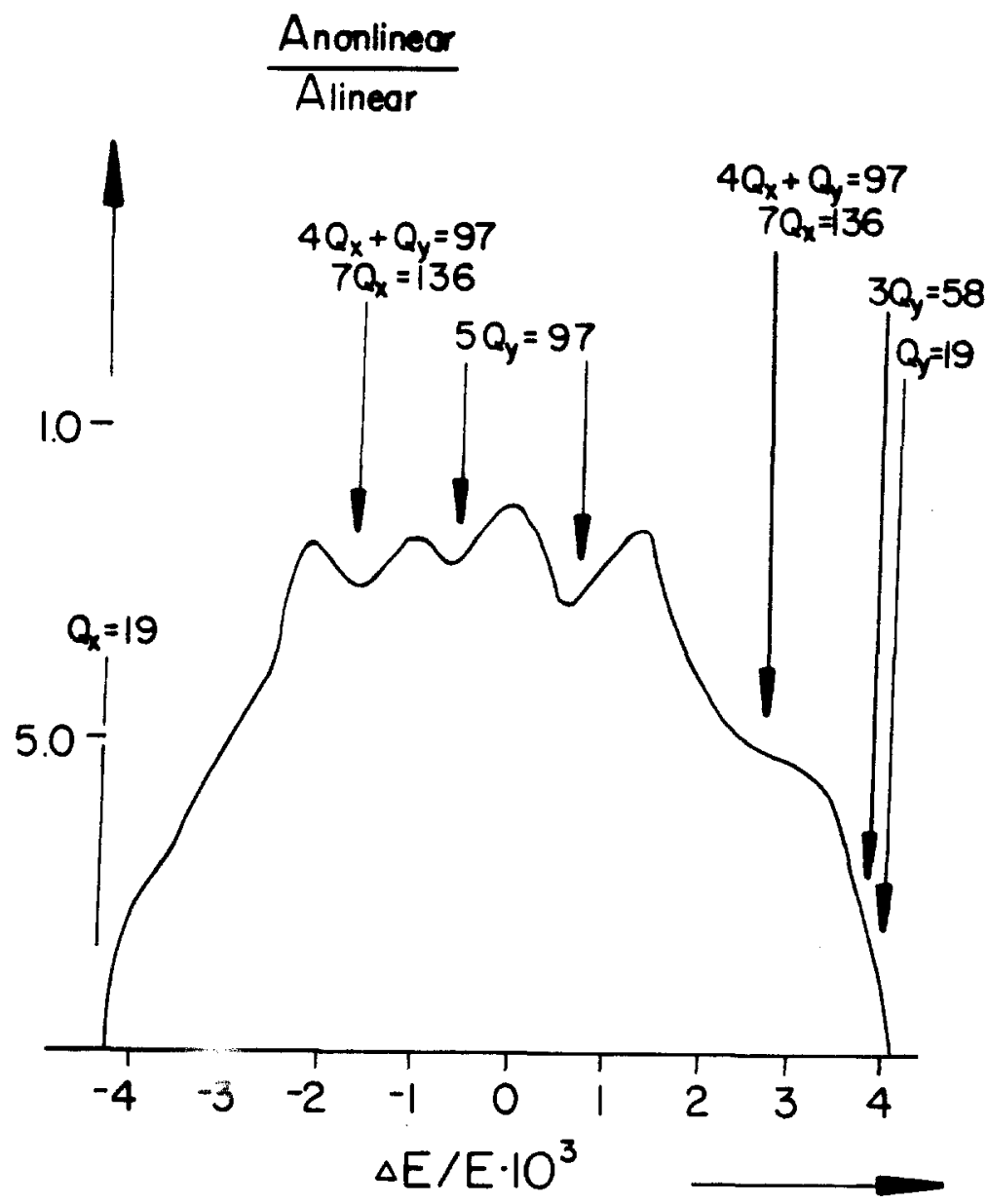

fig 7 Nonlinear Acceptance Reduction for the Extraction Optics versus Energy Deviation

The multipole acceptance shows at $d E / E=+1-0.05 \%$ respectively a dip in acceptance. This can be seen more clearly by plotting the acceptance (elliptical beam) in units of the corresponding linear acceptance (fig 7). The nonlinear tune shifts are plotted versus dE/E in fig 8. The tunes move relative to the resonances as shown in $f i g$. For both fig 8 and fig $9 a$, the tune for zero betatron amplitude is 


\section{$-16-$}

calculated. The tunes of particles with finite betatron amplitudes about the design orbit are shifted by the zero harmonic component of even order multipole fields (quadrupole,octupole etc). Using the formulae given by ref/8/ and applying them to an ideal machine including the averaged multipole components of dipoles, one finds that the only important contribution comes from the octupole term. With same amplitudes for oscillations in $x$ and $y$ the tune shift is given by $d_{x}=-0.0021 \varepsilon / \pi \mathrm{mm} \mathrm{mmr} \mathrm{(} \varepsilon$ : beam emittance). At the acceptance limit the tune shifts are $d_{x}=-0.01$ and $d_{Q_{x}}=0.01$. Off energy there are additional contributions from the strong 14-pole and 18-pole components. All these effects have not been calculated in in detail but if the tune curve in fig 9 is shifted by $d Q_{x}=-0.02$ $d Q_{y}=+0.002$, all dips in fig 7 can be explained by resonance lines. At this point I should mention that Al Russell has Fourier analyzed the betatron oscillations at the acceptance limit. For the TEVATRON 'as built" a similar tune shift is found $/ 9 /$. If multipole fluctuations of multipole fields are introduced in the calculations, this resonance structure becomes from case to case stronger or weaker, but doesn't change qualitatively. However the additional acceptance reduction is drastic and their influence cannot be neglected ( see fig 3 ).

Comparing the acceptance for the TEVATRON 'as built' with the worst of the statistical cases (fig 4) one finds than for small dE/E the acceptance reduction is significantly smaller. Note that the dips in the acceptance plotted versus $d E / E$ have disappeared. There are two reasons for this fact: 
The bending magnets have been installed so that the sextupole driving terms become mimimized. Another reason may be that the details of the statistical distribution reach to the 5 th standard deviation which might be to conservative.

The TEVATRON acceptance has to be compared with the need for acceptance. The measured beam emittance in the TEVATRON is about

$$
\varepsilon=0.23 \pi \mathrm{mm} \mathrm{mrad} \text { at injection energy }(E=150 \mathrm{GeV}) / 6 /
$$

which is more than an order of magnitude smaller than the TEVATRON acceptance. In the high beta straight sections, an amplitude of at least $25 \mathrm{~mm}$ remains stable. This provides sufficient stability for controlled slow extraction. The electrostatic wire septum is at an offset from center of $14 \mathrm{~mm}$. So far the tracking results are in agreement with the experience made with the TEVATRON in the meantime $/ 6 /$.

Introducing closed orbit distortions, one finds that the acceptance will simply be reduced by $A=\left(\sqrt{A \beta_{x}}-x_{c .0}^{r m s}\right)^{2} / \beta_{x}$. From this it may be concluded, that the whole free space in the vacuum chamber is available for closed orbit distortions which also is in agreement with TEVATRON experience. In the low beta case, which is discussed in the following paragraph, the linear acceptance ( solid line fig 5 ) is strongly influenced by the off energy beta beat which causes an increase of acceptance at negative and a decrease at positive energy deviations with respect to the ideal linear acceptance (broken line fig 5 ). The complete breakdown of acceptance occurs when the horizontal tune hits the half integer stopband at $d E / E=$ $+0.225 \%$. Note that the sign of the beta beat follows from the 


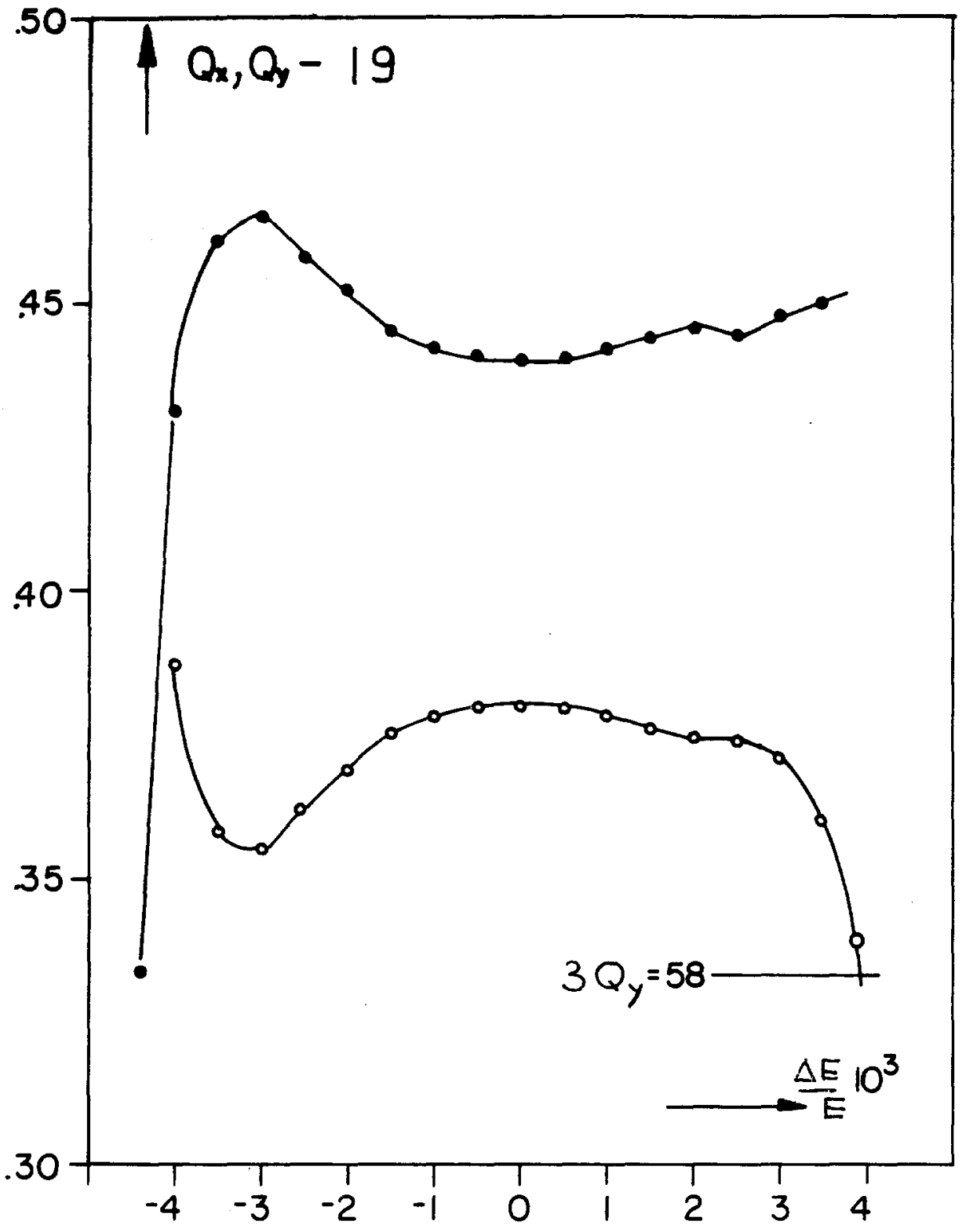

fig 8 Nonlinear Tune Shift for Extraction optics

- Horizontal Tune

- Vertical Tune 

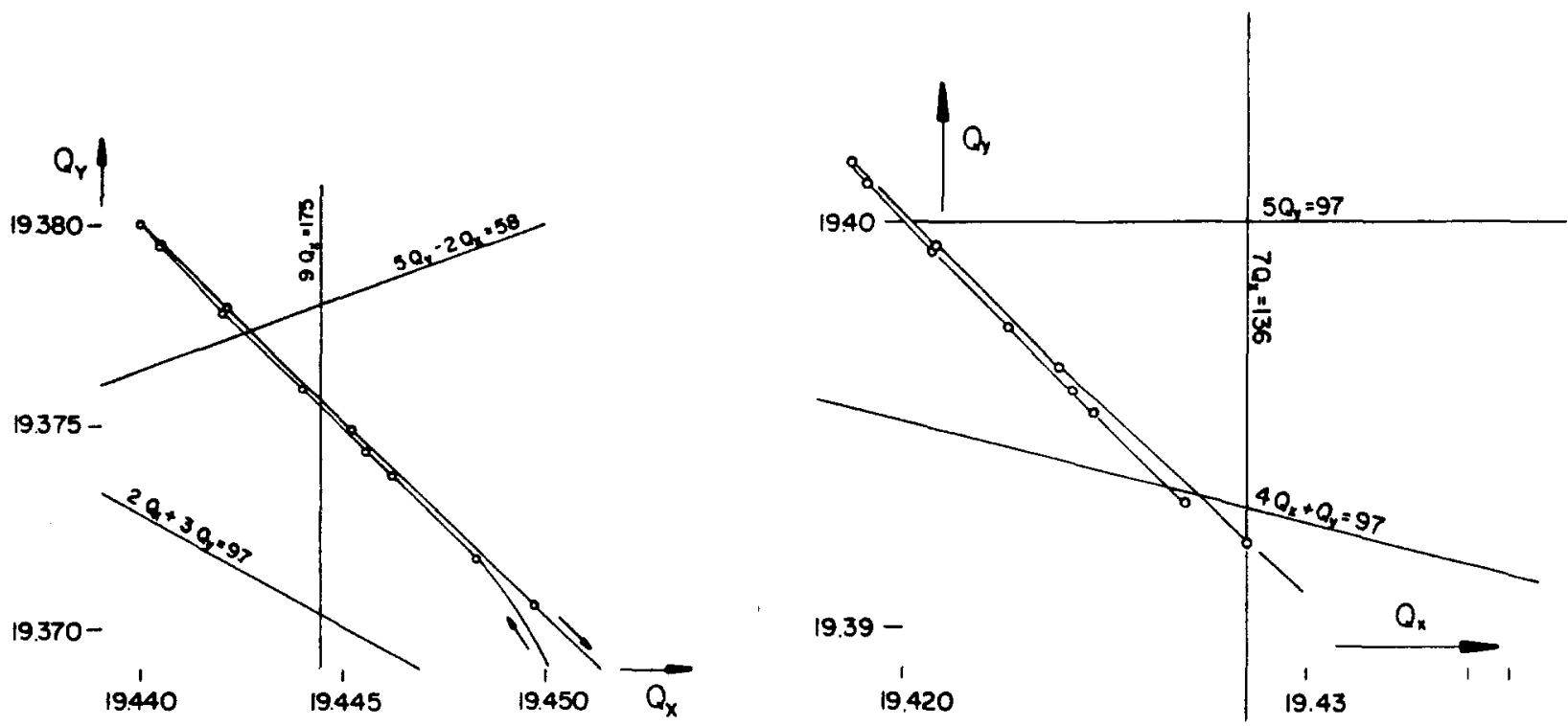

(a)

(b)

fig 9 Nonlinear Tune Shift and Resonance Lines for Extraction optics a) small amplitude tune b) shifted tune (see text) Points correspond to steps in $d E / E=0.5 \%$. The point in the left upper edge is the on energy point.

chosen working point with $Q_{x}=19.565$ and $Q_{y}=19.580$.

Despite the larger chromaticities in the low beta optics $\left(\xi_{x, y}=-29\right)$

the reduction of acceptance due to the sextupole fields needed

for chromaticity compensation is even smaller than for the extraction optios (triangles fig 5). This occurs for mainly two reasons :

The maximum betatron amplitudes in the arcs are only $11.7 \mathrm{~mm}$

(on energy, sextupoles only). Therefore nonlinear fields in the

arc become less important for the acceptance. Moreover because the 
dispersion $D$ is matched to nearly zero at the interaction point, it is no longer periodic in the arc but shows the $\sin ^{2}\left(\phi_{x} / 2\right)$ behavior of the unperiodic dispersion with peak values as twice as much than for the periodic one. This reduces the off energy acceptance by a factor of 1.5 but has the positive effect that only small sextupole fields are needed for chromaticity compensation. This means that the minimum beta functions at the interaction point are not limited by chromatic effects. Thus more than one interaction point or smaller beta functions should be possible from this point of view. Note that the off energy beta beat becomes even smaller when sextupoles are turned on. Thus more than two sextupole families to fight the beta beat are not necessary. The acceptance with multipole fields ( average values in dipole magnets and quadrupole magnets in the low beta straight section, circles in fig 5) has been evaluated (on energy) starting the tracking from different positions in the lattice, among them the maximum beta point in $Q 99\left(\hat{\beta}_{x}=933 \mathrm{~m}\right)$. Contrary to the extraction case acceptance doesn't vary when it is evaluated at different positions. However it is more sensitive to the choice of working point than is the case in the extraction optics. Fig 12 shows the acceptance for various working points. That is because the nonlinear tune shift becomes somewhat stronger (see $f i g$ ) and is influenced by coupling effects.

In order to explain the structure which appears in the acceptance curve in fig 5, the dependence of acceptance on tune is investigated. Because the betatron amplitudes in the dipoles are smaller than in the extraction case, less tune shift is expected. To determine it 

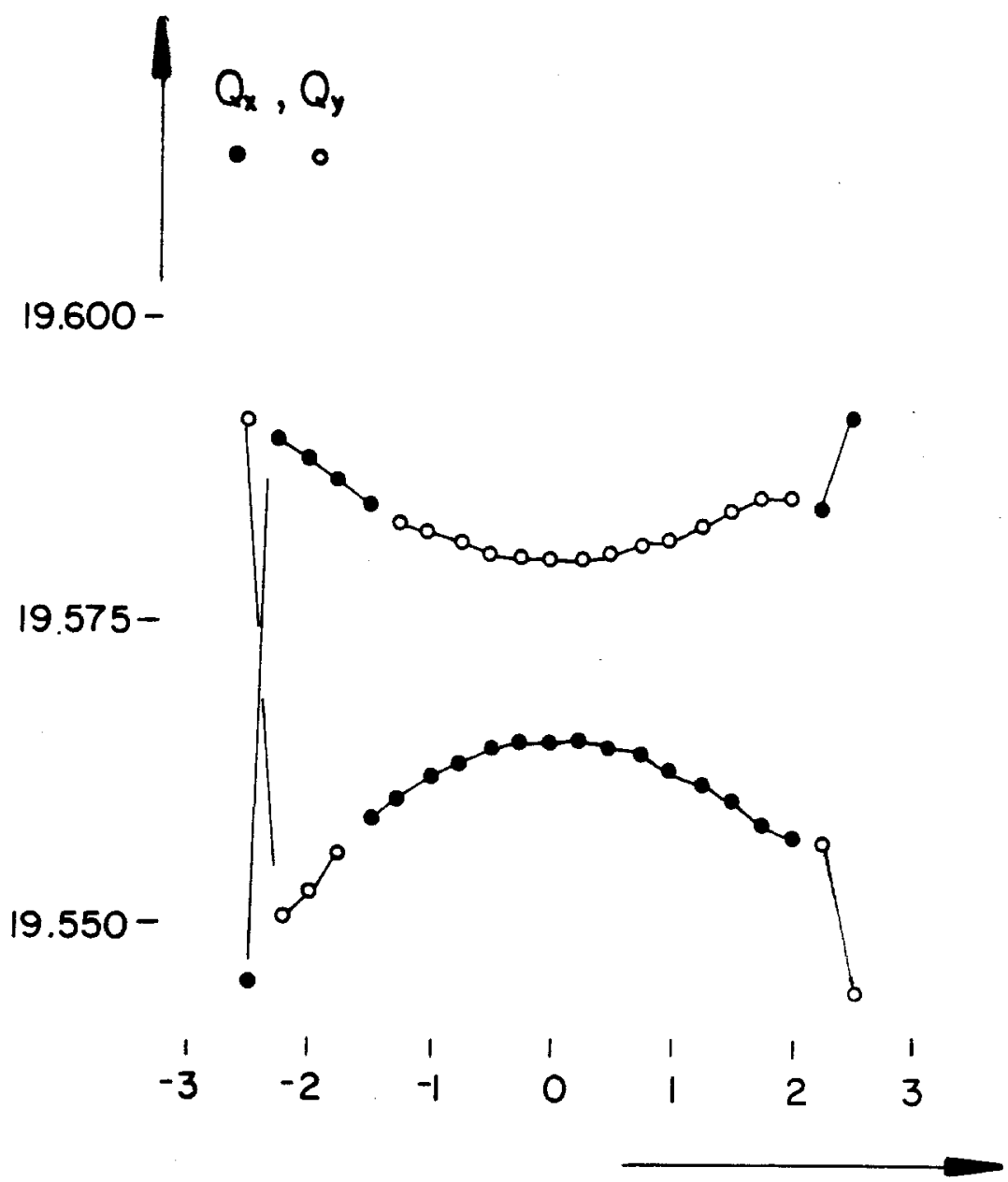

$\Delta E / E \cdot 10^{3}$

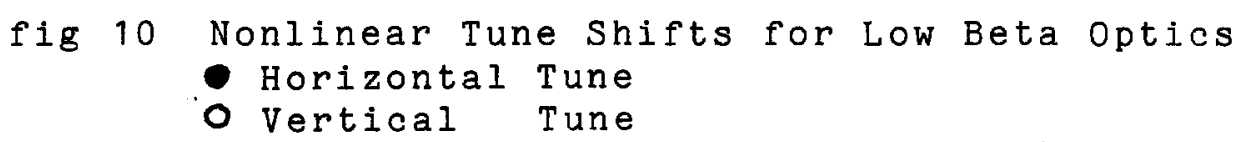

the acceptance is calculated as a function of the tunes near a resonance. Results are shown in fig 11. The acceptance is reduced if the horizontal tune approaches the 7 th order resonances $n Q_{x}+m_{y}$ $=137$. But this dip occurs at a distance of dQ $=0.0026$ from the 


$$
-22-
$$

resonance $7 Q_{x}=137$, from which one derives an amplitude dependent tune shift of $\mathrm{dQ}_{x}=-0.0026$ at $A=0.72 \mathrm{mmm} \mathrm{mrad}$. The amplitude dependence becomes rather large at higher amplitudes which can be seen from the large width of the resonance. It has been verified that this resonance is in fact $7 Q_{x}=137$ by varying the vertical tune and finding the same acceptances. Note that the asymmetric form of the resonance is due to the amplitude dependence of the tune. Approaching the small amplitude tune from above towards the resonance the tune shift decreases because the maximum amplitude is decreased. Therefore the effective tune approaches more slowly the resonance than the small amplitude tune does. The contrary occurs when approaching from below the resonance. The same tune shift but at higher amplitudes and opposite sign is found for the vertical tunes with $\mathrm{dQ}_{y}=+0.0026$ at $A=0.87 \pi \mathrm{mm} \mathrm{mrad}$. The analytical formula gives $d Q_{y}=0.0018$ taking into account the octupole term only. The resulting tune curve in the resonance diagram is shown in fig 12 where points indicate the zero amplitude tune and the broken line is the estimated tune of particles with maximum betatron amplitudes. If this tune shift is taken into account the tune curve hits the resonance $9 Q_{x}=176$ just at $d E / E=-0.15 \%$ where the dip in the acceptance curve occurs (see figures 5 and 12 ). However these results should be considered with care because the off energy tune shift can be very different from the on energy one, especially if the dispersion is as large as in the low beta optics. 


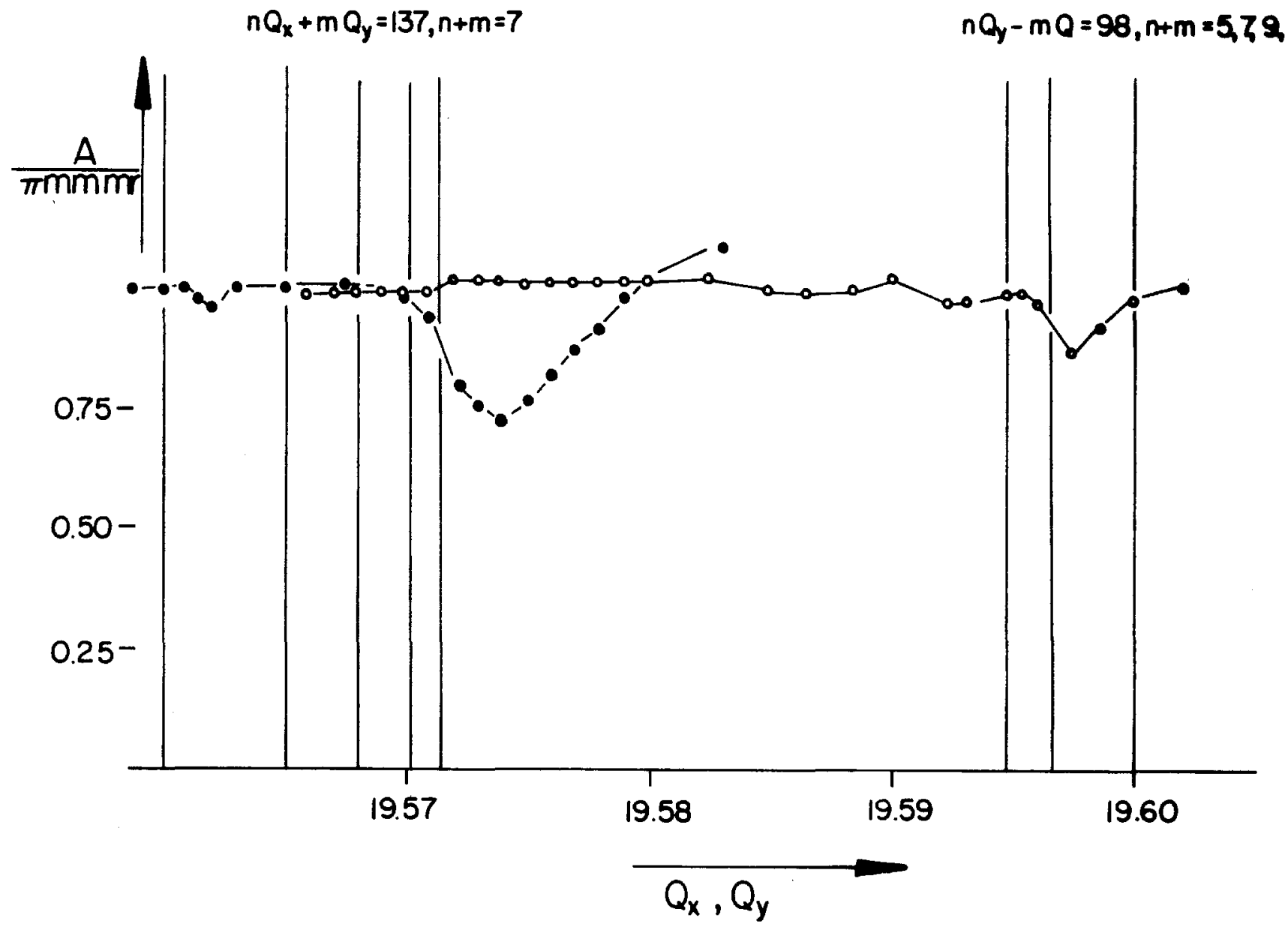

fig 11 Low Beta Acceptance vs Tunes

- $Q_{x}, Q_{y}=19.58$

$\circ Q_{y}, Q_{x}=19.58$

The influence of multipole fluctuations is somewhat smaller for the low beta optics than for the extraction optics ( see full circles in fig 5). The most pessimistic case results in an acceptance $A=0.74 \pi \mathrm{mm}$ mrad. This has to be compared with the maximum beam emittance which can occur. A mismatched injection in the extraction optics at $150 \mathrm{GeV}$ may result in a beam emittance of $\varepsilon=2.6 \pi \mathrm{mm}$ mrad (maximum acceptance for extraction optics). 
After dilution of coherent oscillations and adiabatic incoherent damping, the beam emittance at $1 \mathrm{TeV}$ is $0.39 \pi \mathrm{mm}$ mrad. Thus the remaining place for orbit displacements in the low beta quadrupoles is $7 \mathrm{~mm}$. With the foreseen equipment in the low beta straight section orbit distortions can be corrected at least to $5 \mathrm{~mm} / 7 /$. If the injection is well adjusted, the maximum beam emittance is much smaller. Measurements in the TEVATRON $/ 6 /$ result in an emittance of $\varepsilon=0.23 \pi \mathrm{mm} \mathrm{mrad}$ at $150 \mathrm{GeV}$ and $\varepsilon=0.07 \pi \mathrm{mm} \mathrm{mrad}$ at $520 \mathrm{GeV}$. This is indeed very small compared to the available acceptance.

\section{(4) Conclusion}

In the most pessimistic cases, the TEVATRON acceptance has been determined to be $A=2.6 \pi \mathrm{mm}$ mrad for the extraction case and $A=0.74 \pi \mathrm{mm} \mathrm{mrad}$ for the low beta case for zero energy deviation respectively. The TEVATRON dynamic aperture is $25.6 \mathrm{~mm}$ for the extraction case (worst statistical multipole distribution). The tracking results can be understood at least qualitatively. For the extraction optics, the tracking agrees with the experience which has been gained from TEVATRON performance so far. However special measurements as the experimental determination of the off energy acceptance would be very helpful in the process of getting confidence to the simulations. Tracking results for the low beta optics don't show large evidence for problems in low beta performance.

However it is necessary that nonlinear resonances are avoided by an appropriate choice fo the working point. The orbit distortions have to be small. In the arc they should stay below $2.5 \mathrm{~mm}$ 
and maximum orbit displacements in the low beta quadrupoles have to be restricted to $7 \mathrm{~mm}$, which causes no problem for the control system .

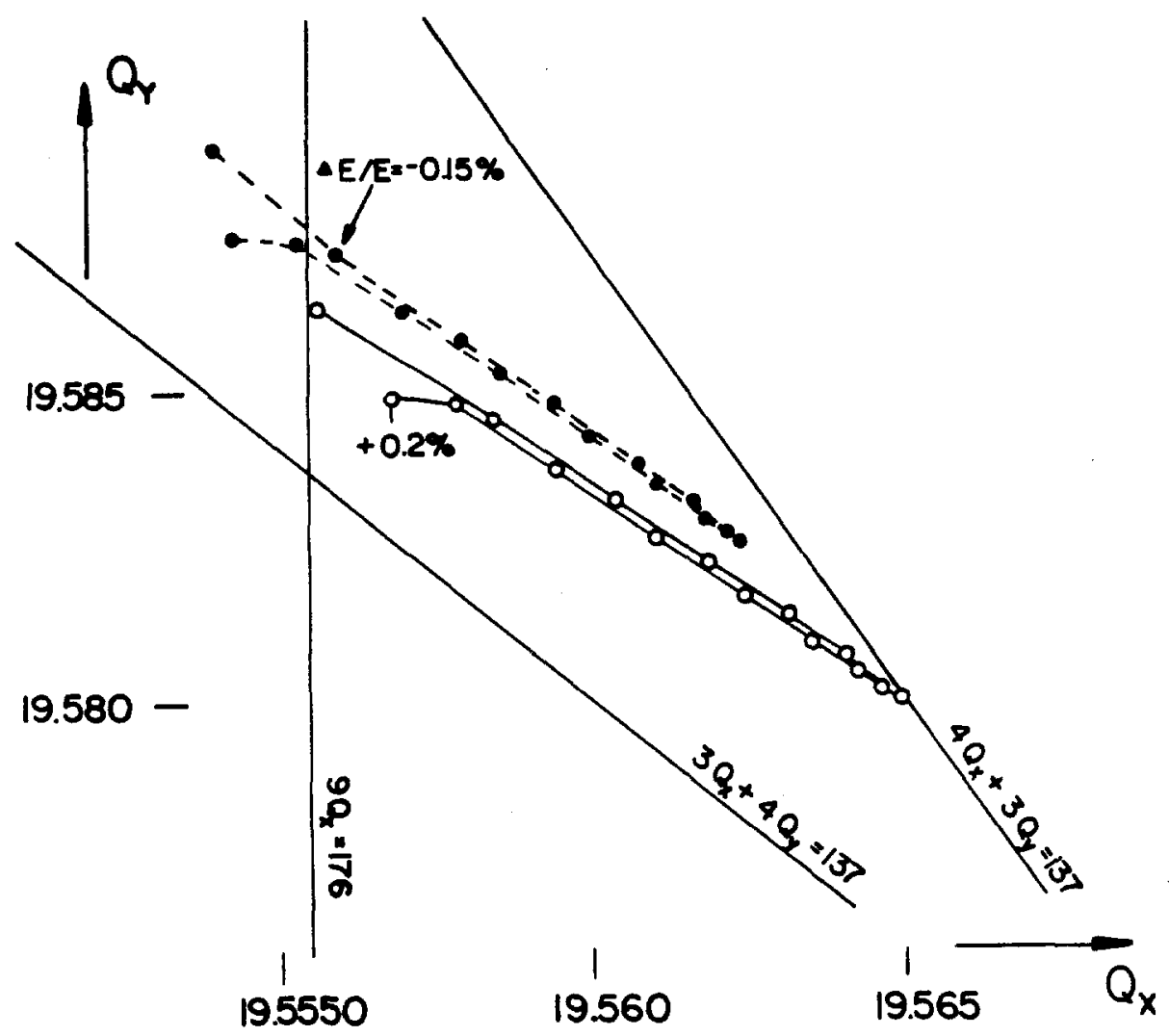

fig 12 Tune Diagram Low Beta Optics

Circles are the zero amplitude tunes at different $d E / E$ which differ by $d E / E=0.025 \%$.

Open Circles : Zero Amplitude Tune

Full Circles : Shipted Tune 


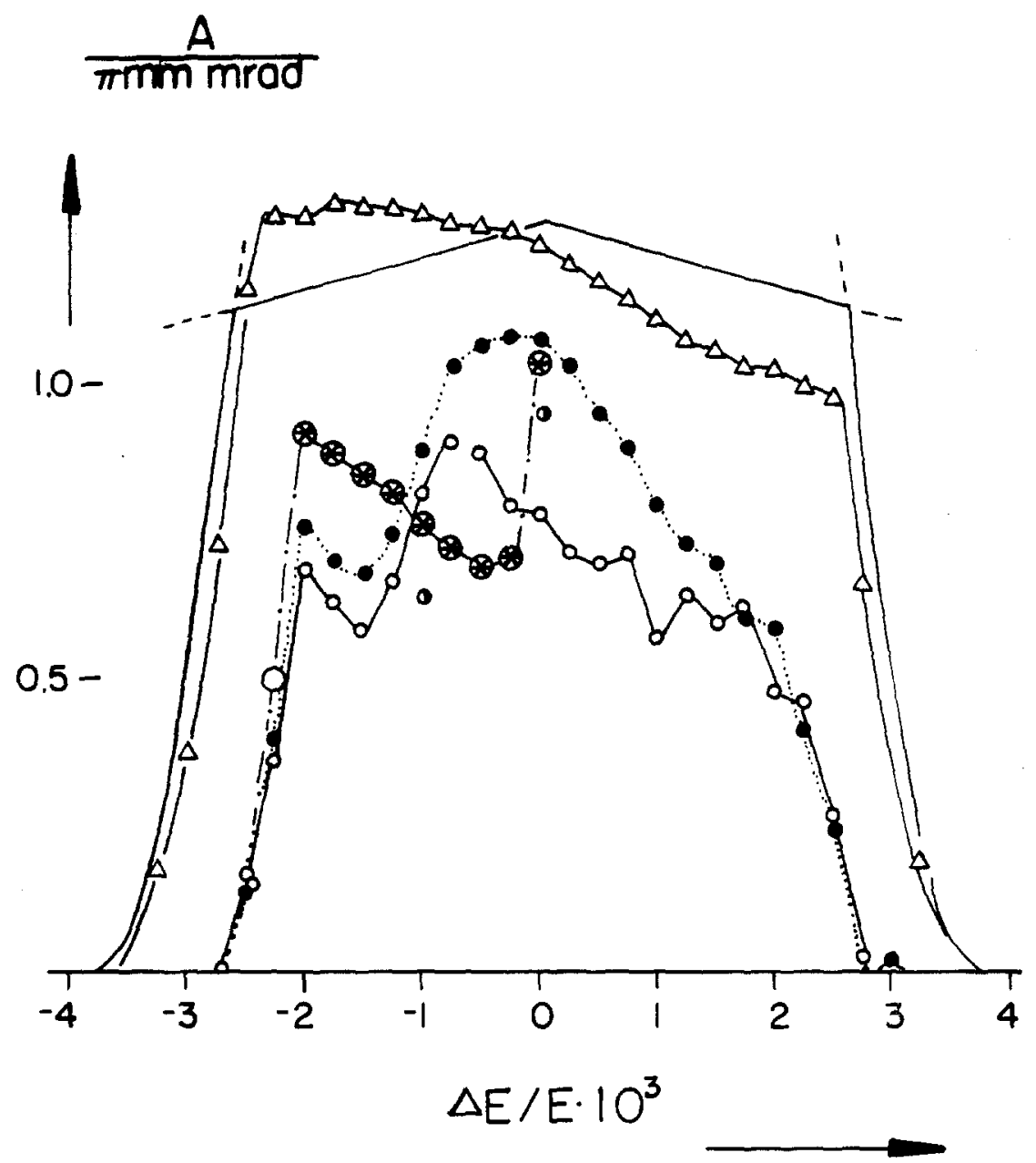

fig 13 Low Beta Acceptance for various working points
- $Q_{x}=19.587, Q_{y}=19.582$
- $Q_{x}=19.565, Q_{y}=19.580$
- $Q_{x}=19.575, Q_{y}=19.589$
- $Q_{x}=19.578, Q_{y}=19.584$ 
Acknowledgment

The author wishes to thank Helen Edwards, Don Edwards and Sho Ohnuma for valuable discussions.

References

/1/ Sho Ohnuma, private communication

/2/N. Gelfand, A.Russell, to be published

/3/ A. Wrulich, Tracking Studies in Hera, DESY HERA 82/07, June 1982

/4/ D.E. Johnson, TEVATRON BO Low Beta Tuning Report, Fermilab TM-1106 1700.000, May 1982

/5/ R. Hanft et al, Magnetic Field Properties of Fermilab Energy Saver Dipoles IEEE Transactions of Nuclear Science Vol NS-30, no4, August 1983

/6/ Betatron amplitudes of $15 \mathrm{~mm}$ and more have been observed during the TEVATRON commissioning by myself and $I$ am sure by other commissioners. It has been caused accidentally e.g. by mismatched injection. However the beam could not be accelerated under such conditions and was generally lost when the ramp has been turned on and the $r f$ power has been increased. During commissioning various measurements have been done so far. Among them the measurement of emittance as quoted above. Beam has been success fully extracted. All these results haven't been published yet.

/7/ H.E. Fisk, Existence Proof for BO Trim Dipole Layout, not published

F. Willeke, Comment on Orbit Correction with steering Dipoles in the Low Beta Case, not published

18/ Sho Ohnuma, Shifts in Betatron Oscillation Frequencies caused by Average Multipole fields

Fermilab, $\bar{p}$ Note 324 , July 1983 